on the left lateral border of the tongue and deviation of the tongue to the left side. She complained of intolerance to hot and spicy food and hoarseness in her voice. Clinical diagnosis was confirmed histopathologically as moderately differentiated squamous cell carcinoma of the tongue.

The aetiology of oral cancer in younger adults remains unclear; however, one needs to ponder on the ubiquitous human papilloma virus (HPV). Oral cancers hidden in cryptic locations of the oral cavity, mimicking symptoms in the initial stages, such as those of pericoronitis and tonsillitis, often confront clinicians with the daunting task of managing them in advanced stages. Thinking outside the box with an early diagnosis and swift referral is key before these tumours progress. In this patient, an early marriage followed by early coitus might have triggered the dormant HPV. ${ }^{1}$ HPV-induced oral cancer known for its demographic shift may be an emerging and distinct clinical entity, although future research is necessary.

Sameep S. Shetty, Premalatha Shetty, Mangalore

1. Rotkin I D. Relation of adolescent coitus to cervical cancer risk. JAMA 1962; 179: 486-491.

DOI: 10.1038/sj.bdj.2016.206

\section{CONTACT DERMATITIS}

\section{Fiddler's neck}

Sir, dermatoses in musicians can occur in players of brass, percussion, wind instruments and also players of string instruments. ${ }^{1}$ We present a 36-year-old woman (Fig. 1) who plays the violin with an irritant area from contact of the chin rest with the left submandibular neck who came for routine dental examination. Cutaneous examination of the left submandibular neck demonstrated a hyperpigmented, erythematous plaque. A panoramic radiograph showed some calcifications and one of them is an oval calcification with smooth periphery and has the appearance of laminations, giving it a bull'seye or targetoid appearance in the internal

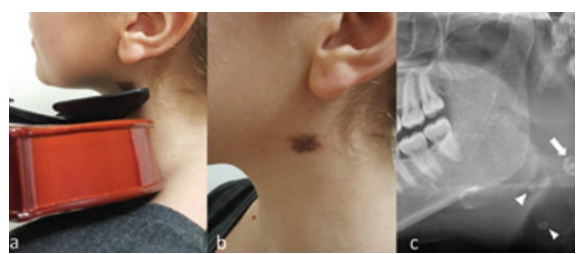

Fig. 1 a) Touchpoint of violin on left submandibular area; b) Irritant area from contact of the violin; c) Targetoid shaped oval calcification on panoramic image (white arrow), other small calcifications (white arrowheads) aspect like phlebolith.

One of the most common types of traumainduced dermatitis in musicians is fiddler's neck syndrome, induced by violins and violas. There is greater variety of clinical aspects in this syndrome than in the usual irritant contact dermatitis. The lesions can be localised plaques of erythema, sometimes pigmented or lichenified. ${ }^{2}$ However, these lesions are generally not considered radiographically and the direct relationship between the violin and phlebolith-like calcification has never before been recorded in medical literature. In some literature violin pressure points have been shown to be among the causes of dystrophic calcification. ${ }^{3}$

We evaluated this calcification seen in the neck as phlebolith from an acquired vascular malformation or a dystrophic calcification. An increase in the number of cases like this would help us to understand this condition more clearly.

\section{G. Geduk, Turkey}

1. Caero J E, Cohen P R. Fiddler's neck: Chin restassociated irritant contact dermatitis and allergic contact dermatitis in a violin player. Dermatol Online J 2012; 18: 10.

2. Crepy M N. Skin diseases in musicians. Eur J Dermatol 2015; 25: 375-383.

3. Weedon D. 14 - Cutaneous deposits. In Weedon D (ed). Weedon's Skin Pathology, 3rd ed. pp 369-396. Edinburgh: Churchill Livingstone, 2010.

DOI: 10.1038/sj.bdj.2016.207

\section{TMD}

\section{Costen's Syndrome}

Sir, it was most interesting to read the short summary 'Revisiting Costen Syndrome' (BDJ 2016; 220: 15).

Costen's Syndrome has been largely ignored over the years since it was first reported in 1934. Research into the embryology of the temporomandibular joint has shown that the middle ear and the joint are closely related. The disco-malleolar ligament, a remnant of the Meckel's cartilage, connects the malleus to the retro-discal tissue of the joint. It should, therefore, not be surprising that aural and joint symptoms are closely linked.

Perhaps it is now time for Costen's early work to be reconsidered and his contribution to multi-disciplinary work recognised.

R. M. Dean, by email DOI: 10.1038/sj.bdj.2016.208

\section{PERIODONTOLOGY}

\section{The value of tooth and cocktail picks}

Sir, as a registered specialist in periodontology I concur that toothbrushing is probably the most important and common oral hygiene practice; however, it does not provide adequate interdental hygiene. Originally, dental woodsticks were advocated by the dental profession as 'gum massagers' to be used to massage inflamed gingival tissue in the interdental areas to reduce inflammation and encourage keratinisation of the gingival tissue. ${ }^{1}$

It has been shown that a triangular, pointed woodstick inserted interdentally can maintain a plaque-free region 2-3 mm subgingivally. ${ }^{2}$ For open interdental spaces, common among adults, woodsticks seem most appropriate. ${ }^{3}$ In periodontitis patients, the woodstick will depress the papilla which may help in recontouring the interdental tissues and consequently preclude the need for periodontal surgery. ${ }^{4}$ Woodsticks have the advantage of being easy to use and can be used anywhere. ${ }^{1}$

Round, pointed-end toothpicks or cocktail sticks are an undervalued resource in daily plaque control in the $45 \%$ of the population that are susceptible to chronic periodontitis. In the past ten years, I have found them to be effective during the treatment stages and in the maintenance phase in patients diagnosed with moderate/advanced periodontal disease, especially recalcitrant cases. The tip is ideally shaped for access to all root morphologies and most furcations. I have also found them to have the ideal profile for use around the circular emergence profile of dental abutments and dental implants.

In most cases patients have described to me that this was mento-physically less demanding than the other available interdental aids they had previously used. Having seen the success that these toothpicks achieved, I introduced their use in a YouTube video entitled 'Oral hygiene instructions with gum specialist Dr Hafeez Ahmed'. It frees up clinical time and offers the patient a resource for ongoing reminders and training.

So far, there are no high quality prospective studies on the round pointed end tooth pick or the cocktail stick to confirm that they are an effective interdental device in reducing the clinical parameters of plaque and gingival inflammation but I am hopeful that this letter might encourage this.

H. Ahmed, by email

1. Galgut P N. The need for interdental cleaning. Dent Health 1991; 30: 8-11.

2. Morch T, Waerhaug J. Quantitative evaluation of the effect of toothbrushing and toothpicking. J Periodontol 1956; 27: 183-190.

3. Axelsson P. Mechanical plaque control. In Lang N P, Karring T. Proceedings of the 1st European Workshop on Periodontology, Thurgau Switzerland. p 225. Quintessence, 1994.

4. Bear P N, Morris M L. Textbook of periodontics. p 159. Philadelphia, USA: JB Lippincott Company, 1977.

DOI: 10.1038/sj.BDJ.2016.209 